\title{
Prognostic Significance of Initial $r$-Wave in Leads VI and aVR Before PTSMA in Patients with Hypertrophic Obstructive Cardiomyopathy
}

\author{
Xiaowei Jiang $\mathbb{D}^{1,2}$ \\ Shubin Qiao ${ }^{2}$ \\ 'Cardiology Department, Xiangya \\ Hospital, Central South University; \\ National Clinical Research Center for \\ Geriatric Disorders, Xiangya Hospital, \\ Central South University, Changsha, \\ Hunan, People's Republic of China; ${ }^{2}$ State \\ Key Laboratory of Cardiovascular \\ Disease, Fuwai Hospital, National Center \\ for Cardiovascular Diseases, Chinese \\ Academy of Medical Sciences and Peking \\ Union Medical College, Beijing, 100037, \\ People's Republic of China
}

Correspondence: Shubin Qiao

State Key Laboratory of Cardiovascular Disease, Fuwai Hospital, National Center for Cardiovascular Diseases, Chinese Academy of Medical Sciences and Peking Union Medical College, Beijing, I00037,

People's Republic of China

Email qsbfw@sina.com
Objective: Percutaneous transluminal septal myocardial ablation (PTSMA) is one of the septal reduction therapies without all satisfactory results in hypertrophic obstructive cardiomyopathy (HOCM) patients. Initial r-wave in leads V1 and aVR on the surface electrocardiography is the sign of ventricular septal base depolarization. Whether the initial r-wave in leads V1 and aVR is predictive of good mid-term response to PTSMA in patients with HOCM remains unknown.

Design, Settings, and Participants: Among 546 consecutive patients evaluated in Fuwai Hospital (Beijing, China), we selected HOCM patients who underwent PTSMA. During the 3-year follow-up after PTSMA, primary outcomes included death, surgical myectomy, syncope, and NYHA classification III/IV.

Results: At last, 85 patients were selected, and were assigned to 3 groups based on the presence of initial r-wave in leads V1 and aVR. Through multivariable Cox and KaplanMeier method, patients with initial r-wave in both leads V1 and aVR were independently associated with a decreased risk of primary outcomes (HR: $0.112 ; 95 \%$ CI: 0.024 to 0.518 ; $p=0.005$ ) during 3-years' follow-up.

Conclusion: The results suggest that the presence of initial r-wave in both leads V1 and aVR on the surface 12-lead ECG is highly predictive of good mid-term response to PTSMA in patients with HOCM.

Keywords: hypertrophic obstructive cardiomyopathy, percutaneous transluminal septal myocardial ablation, electrocardiography

\section{Introduction}

Hypertrophic cardiomyopathy (HCM) is a common heritable disease with a prevalence of 1 in 500 of the general population. ${ }^{1}$ Asymmetric septal hypertrophy without an alternative cause is the most common manifestation of this disorder, and $70 \%$ of the patients with HCM have associated left ventricular outflow tract (LVOT) obstruction. ${ }^{2}$ Percutaneous transluminal septal myocardial ablation (PTSMA) is one of the septal reduction therapies that relieve symptoms related to heart failure in patients with hypertrophic obstructive cardiomyopathy (HOCM). ${ }^{3,4}$ However, satisfactory results were not observed in all patients who underwent PTSMA. ${ }^{3,5}$ It is important to identify the determinants of PTSMA outcome before PTSMA procedure for better selection of patients and potential modification of treatment plan in an individual patient.

Septal reduction via PTSMA alters ventricular activation as well as causes a localized myocardial infarction (MI). ${ }^{6}$ Altered septal activation by DDD pacing 
from the RV has been shown to reduce the outflow tract pressure gradient. ${ }^{7}$ These studies indicated that early activation of ventricular septal disturbance induced by procedure could alleviate symptoms and outflow tract gradient. Electrocardiography (ECG) is a non-invasive examination method that is widely used in patients with HOCM. Cardiac activity was noted first in the upper septal region, 8,9 and depolarization then propagates in a base-toapex sequence. ${ }^{10}$ Lead aVR is located in the upper right quadrant and reflects the electrical activity at the ventricular septal base. The initial r-wave in lead V1 represents initial ventricular activation, and the initial r-wave in lead aVR represents ventricular septal base activation. Whether the appearance of the initial $r$-wave in V1 and aVR could predict the midterm response to PTSMA is unknown.

\section{Method}

This study was approved by the Ethics Committee of Fuwai Hospital and performed following the Declaration of Helsinki. All participants provided their written informed consent to agree with using their clinical information.

\section{Study Population and Study Design}

We analyzed retrospectively consecutive patients with drug-refractory HOCM that were treated with PTSMA for the first time in Fuwai Hospital (Beijing, China), between June 2012 and January 2015. All subjects had undergone comprehensive clinical, physical examination, 12-lead electrocardiography, echocardiography, coronary angiography, and cardiac magnetic resonance (CMR) within 1 week before PTSMA. The diagnosis of HCM was based on a maximum LV wall thickness no more less $15 \mathrm{~mm}$ (or no more less $13 \mathrm{~mm}$ with an unequivocal family history of HCM), as measured by echocardiography or CMR, excluded other cardiac or systemic diseases capable of producing such magnitude of hypertrophy. ${ }^{11}$ In all patients, the diagnosis HOCM was defined as HCM patients with LVOT pressure gradients $\geq 30 \mathrm{~mm} \mathrm{Hg}$ at rest or during provocation by the Valsalva maneuver, and confirmed by clinical and noninvasive criteria. Among a total of 546 patients with HCM, we selected HCOM patients with underwent the PTSMA procedures. Patients were excluded from analysis if they had undergone PTSMA in other hospitals before enrollment $(n=2)$, right bundle branch block (RBBB) before PTSAM ( $n=1)$, WolfParkinson-White (WPW) $(\mathrm{n}=2)$, uncompleted PTSMA procedure $(n=3$, due to occurred with ST-segment elevation in leads II, III and aVF in electrocardiograph monitoring during PTSAM procedure), death for periprocedural complication (retroperitoneal hematoma, $n=1$ ), or lost to follow-up $(n=5)$. Eighty-six patients were included in our study. Among these, patients were classified according to the presence of initial r-wave in leads V1 and aVR within 1 week before the PTSMA procedure, we divided these patients into three groups: Group A defined as the initial r-wave in both leads V1 and aVR, $n=24$; Group B defined as initial r-wave in lead V1 but not in lead aVR, $\mathrm{n}=36$; and Group $\mathrm{C}$ defined as without initial $\mathrm{r}$-wave in both leads V1 and aVR, $\mathrm{n}=35$ (Figure 1). Only one patient with an initial r-wave in lead aVR and without initial r-wave in lead V1 was excluded. The data of 85 patients' baseline were collected from electronic patient records, and clinical outcomes of these patients with HOCM after PTSMA were assessed by outpatient service or telephone interview every half of the year during the 3-year follow-up after PTSMA.

\section{ECG}

Within 7 days before the PTSMA procedure, 12-lead ECG's were performed using Mortara ELI 250 and ELI 280 electrocardiographs with a gain setting of $10 \mathrm{~mm} / \mathrm{mv}$, a paper speed of $25 \mathrm{~mm} / \mathrm{sec}$, a high pass filter of $0.05 \mathrm{~Hz}$, a low-pass filter of $150 \mathrm{~Hz}$, and a notch filter of $60 \mathrm{~Hz}$. Patients with electrocardiographic evidence of prior MI, or right bundle branch block (RBBB), or WPW were excluded. We chose the r-wave in leads V1 and aVR, not the septal q-wave in leads V3-V6, as the presence of septal activation in HOCM patients, because of the clockwise rotation of heart due to obvious ventricular hypertrophy and the activation of the interventricular septal base. The presence of the initial r-wave in leads V1 or aVR was defined as the voltage no less than $0.1 \mathrm{mV}$. All ECGs were reviewed by two investigators. Differences relating to the presence or absence of diagnostic criteria were resolved by consensus.

\section{Echocardiography}

All transthoracic echocardiography was performed, according to the American Society of Echocardiography's recommendation, ${ }^{12}$ using an iE 33 Color Doppler Ultrasound System (Philips Healthcare, Andover, MA). The peak velocity across the left ventricular outflow tract (LVOT) was also measured, and the peak pressure gradient was estimated using the simplified Bernoulli equation, while the LVOT gradient after provocation (Valsalva maneuver) was only 


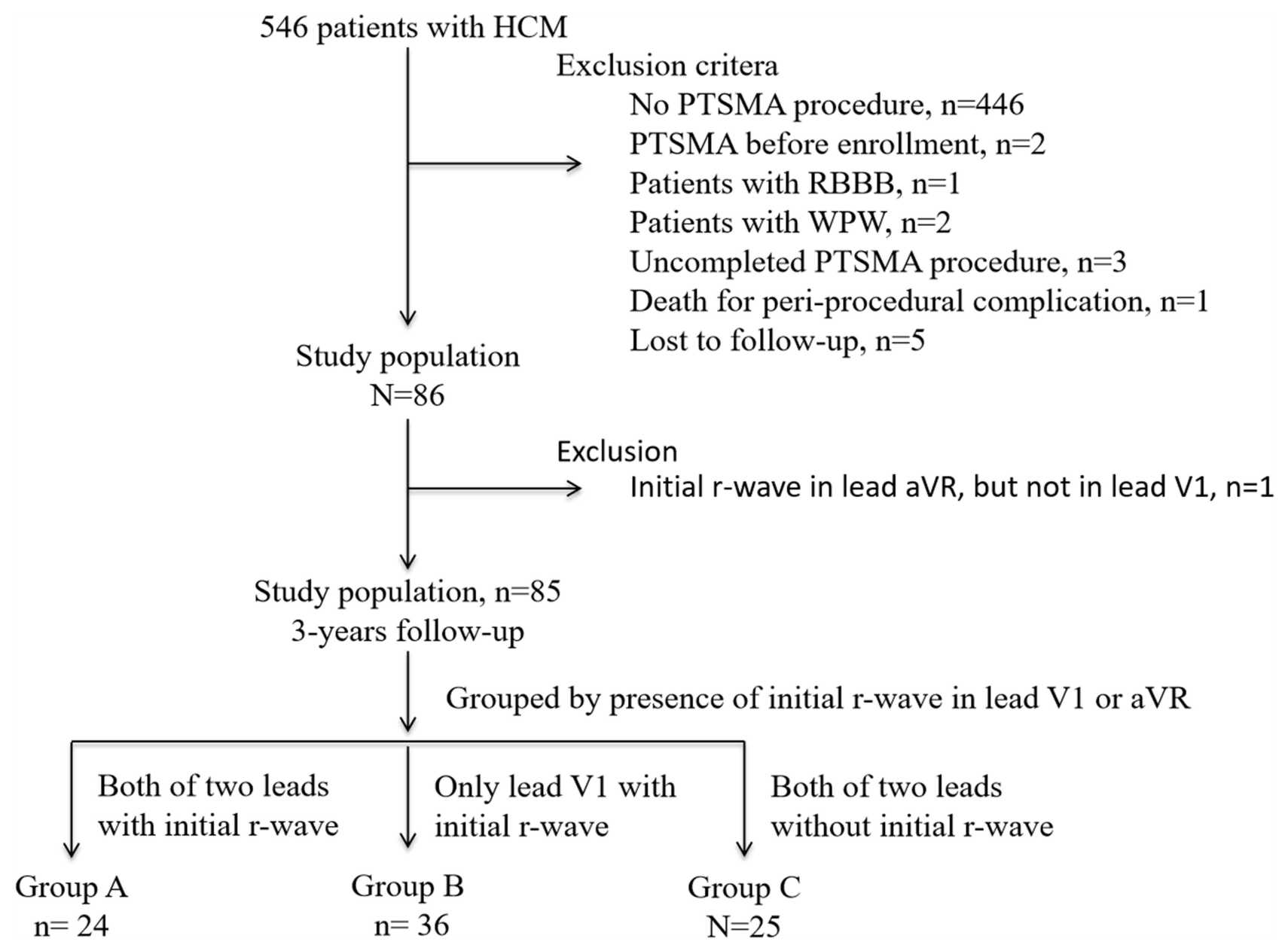

Figure I Study flow.

Abbreviations: HCM, hypertrophic cardiomyopathy; PTSMA, percutaneous transluminal septal myocardial ablation; RBBB, right bundle branch block; WPW, WolfParkinson-White.

determined in those with a resting LVOT gradient less than 30 $\mathrm{mmHg}$.

\section{CMR}

CMR imaging was performed with electrocardiographic gating and breath-holding on a 1.5-T scanner (Magnetom Avanto; Siemens Medical Solutions, Erlangen, Germany). Briefly, ${ }^{13}$ cine images were obtained in left ventricular two-chamber and four-chamber long-axis views, LVOT view, and LV short-axis views by true fast imaging by steady-state precession (TrueFisp) sequence. CMR images were analyzed by an experienced radiologist on a workstation (Siemens Medical Systems, Erlangen, Germany). Endocardial and epicardial contours of the left ventricular myocardium, except papillary muscles, were traced at end-diastole and end-systole on each LV shortaxis cine image by the radiologist. The interventricular septum (IVS), left ventricular end-diastolic volume
(LVEDV), left ventricular end-systolic volume (LVESV), left ventricular ejection fraction (LVEF), stroke volume (SV), cardiac output (CO), and LV mass (LVM) were then calculated in a standard fashion. LVM was derived by multiplying left ventricular myocardial volume measured at end-diastole with the specific gravity of the myocardium $(1.05 \mathrm{~g} / \mathrm{mL})$. All parameters were indexed to body surface area, except LVEF.

\section{PTSMA Indications and Procedure}

The indication for PTSMA included the presence of severe drug-refractory symptoms (NYHA class III/IV, or NYHA class II symptoms with recurrent exercise-induced syncope) in combination with LVOT pressure gradients (PG) $\geq 30 \mathrm{~mm} \mathrm{Hg}$ at rest or $\geq 50 \mathrm{~mm} \mathrm{Hg}$ during provocation by the Valsalva maneuver. The procedure was offered to patients with refractory symptoms despite being administered maximally tolerated medical therapy, and those 
who were not candidates for surgical myectomy due to either the presence of comorbidities (myocardial infarction, valvular disease, or other structural heart diseases) or patient preference. In brief, all patients had a prophylactic temporary pacemaker inserted before the procedure. A 6 Fr femoral arterial sheath was inserted for the guide catheter system and a 6 Fr radial sheath was inserted for pigtail catheter placement into the LV cavity. Continuous invasive peak-to-peak gradients were measured across the LVOT by comparing the peak LV and aortic pressure. A septal perforating artery supplying the obstructing part of the septum was identified on coronary angiography, and was chosen as the target vessel. After the occlusion of the septal target branch by an over-the-wire balloon (Medtronic, Inc, Minneapolis, MN, USA). About $2 \mathrm{~mL}$ of echocardiography contrast medium (SonoVue; Bracco Imaging BV, Geneva, Switzerland) was injected through the balloon catheter lumen under continuous echocardiographic imaging to identify the part of the myocardium that was supplied by the targeted septal artery. After all the patients received morphine chloride intravenously for pain control, $1-4 \mathrm{~mL}$ ethanol was slowly injected into the target vessel through a balloon catheter. The balloon was deflated and removed after $15 \mathrm{~min}$. The occlusion of the target vessel was verified angiographically at the end of the procedure. Acute success was defined as more than $50 \%$ reduction of invasive gradient pre-PTSMA, or less than $30 \mathrm{mmHg}$ of invasive gradient post-PTSMA. All the patients were monitored in the coronary care unit after the procedure for at least 48 hours. Within 7 days after PTSMA, all the patients had been performed transthoracic echocardiography for measuring residual gradient.

\section{Follow-Up}

We defined the primary outcomes included that death, surgical myectomy, syncope, and NYHA classification III/IV. There were no patients who occurred with death, syncope, pacemaker, or stroke during 3-year follow-up after PTSMA. The NYHA functional capacity classification was evaluated as demonstrated according to the 1994 New York Heart Association Classification system. ${ }^{14}$ We collected these events from hospital records and telephone interviews.

\section{Statistical Analysis}

Continuous variables are expressed as mean \pm SD. Categorical variables are shown as frequencies (percentages). The baseline characteristics among the three groups were analyzed by ANOVA and LSD $t$-test for parametric variables, and the chi-square tests or Fisher exact tests for categorical variables. Survival estimates were calculated by the Kaplan-Meier method and the Log rank test was used for comparison. The association between initial r-wave in leads V1 and aVR on 12-lead ECG before PTSMA and outcomes of HOCM after PTSMA were estimated with univariate and multivariate Cox proportional hazards regression models. Multivariable models were fitted using backward elimination of nonsignificant factors at a $10 \%$ level. These covariables in multivariate analysis were mainly selected for the following reasons: the variable which has significance in univariable analysis, or the known variable as related to the outcomes. Model 1 was unadjusted; Model 2 was adjusted with age, sex, and NYHA class III/IV; Model 3 was adjusted with age, sex, NYHA class III/IV, hypertension, NT-proBNP, SAM, and left ventricular mass index. A 2-tailed $p$-value $<0.05$ was considered statistically significant. Statistical analysis was performed with the statistical package GraphPad Prism 5 and SPSS 20.0 (SPSS Inc, Chicago, Illinois).

\section{Results}

\section{Baseline Characteristics}

Baseline clinical and procedural characteristics are summarized in Table 1. Among the total 85 patients, patients were $51.4 \pm 9.7$ years old and consisted of $54.1 \%$ men. A total of $43.5 \%$ of patients presented with hypertension, $60.1 \%$ had NYHA classification III/IV, and 88.2\% had SAM phenomenon in echocardiographic. There were 24 patients with initial r-wave in both leads V1 and aVR, 36 patients with initial r-wave in lead V1 but not in aVR, and 35 patients without initial r-wave in both leads V1 and aVR. Subjects with initial $r$ wave in QRS in both leads V1 and aVR showed lower NT-proBNP $(\mathrm{p}<0.05)$, lower SAM phenomenon $(\mathrm{p}<0.05)$, and lower left ventricular mass index $(\mathrm{p}<0.05)$.

\section{PTSMA Procedure-Related Profiles}

In Table 2, the rate of acute success of PTSMA, with using mean $2.1 \pm 0.8 \mathrm{~mL}$ ethanol, was $87.1 \%$ in all 85 patients, and there were significant differences when grouped by the rate of changes of the presence of initial r-wave in lead V1 or aVR, but none differences in aspect of ethanol volume, acute success rate, and residual gradient according to these three groups. As illustrated in Figure 2, when compared 
Table I Clinical Feature of HOCM Patients Grouped by Initial $r$ in QRS Waves of Leads VI and aVR

\begin{tabular}{|c|c|c|c|c|c|}
\hline & $\begin{array}{l}\text { Total Population } \\
\qquad(\mathrm{n}=85)\end{array}$ & $\begin{array}{c}\text { Group } \\
\text { A }(n=24)\end{array}$ & $\begin{array}{c}\text { Group } \\
B(n=36)\end{array}$ & $\begin{array}{c}\text { Group } \\
C(n=25)\end{array}$ & $p$ value \\
\hline \multicolumn{6}{|l|}{ Electrocardiography } \\
\hline Initial $r$ in QRS wave of lead VI, $n(\%)$ & $60(70.6)$ & $24(100 \%)$ & $36(100 \%)$ & $0(0.0)$ & - \\
\hline Initial $r$ in QRS wave of lead aVR, $n(\%)$ & $24(28.2)$ & $24(100 \%)$ & $0(0.0)$ & $0(0.0)$ & - \\
\hline Age (years) & $51.4 \pm 9.7$ & $52.6 \pm 10.6$ & $52.8 \pm 8.7$ & $48.4 \pm 9.8$ & 0.175 \\
\hline Male, n (\%) & $46(54.1)$ & $13(54.2)$ & $21(58.3)$ & $12(48.0)$ & 0.728 \\
\hline Heart rate (bpm) & $70.9 \pm 13.1$ & $72.0 \pm 12.5$ & $68.3 \pm 12.0$ & $73.6 \pm 14.9$ & 0.271 \\
\hline $\mathrm{SBP}(\mathrm{mmHg})$ & $116.9 \pm 19.5$ & $1 \mid 3.1 \pm 18.0$ & $1 \mid 9.1 \pm 21.4$ & $117.2 \pm 18.3$ & 0.506 \\
\hline $\mathrm{DBP}(\mathrm{mmHg})$ & $72.2 \pm 11.7$ & $70.7 \pm 9.7$ & $72.6 \pm 11.4$ & $73.1 \pm 13.9$ & 0.745 \\
\hline Hypertension, n (\%) & $37(43.5)$ & $9(37.5)$ & $21(58.3)$ & $7(28.0)$ & 0.049 \\
\hline Diabetes mellitus, $\mathrm{n}(\%)$ & $5(5.9)$ & $2(8.3)$ & $3(8.3)$ & $0(0.0)$ & 0.382 \\
\hline Hypercholesterolemia, n (\%) & $29(34.1)$ & $9(37.5)$ & $15(4 \mid .7)$ & $5(20.0)$ & 0.197 \\
\hline Current smokers, n (\%) & $30(35.3)$ & $8(33.3)$ & $15(4 \mid .7)$ & $7(28.0)$ & 0.532 \\
\hline NYHA classification III/IV, n (\%) & $51(60.0)$ & $15(62.5)$ & $15(4 \mid .7)$ & $21(84.0)$ & 0.004 \\
\hline Family history of HOCM, n (\%) & $17(20.0)$ & $4(16.7)$ & $4(I I . I)$ & $9(36.0)$ & 0.075 \\
\hline Family history of SCD, n (\%) & $9(10.6)$ & $2(8.3)$ & $2(5.6)$ & $5(20.0)$ & 0.228 \\
\hline Syncope, n (\%) & $22(25.9)$ & $9(37.5)$ & $7(19.4)$ & $6(24.0 .0)$ & 0.285 \\
\hline Atrial fibrillation, n (\%) & $6(7.1)$ & $0(0.0)$ & $5(13.9)$ & I (4.0) & 0.120 \\
\hline Non-sustained VT, n (\%)* & II (I2.9) & $3(12.5)$ & $3(8.3)$ & $5(20.0)$ & 0.282 \\
\hline NT-proBNP (fmol/mL)* & $152 \mid .3 \pm 1084.6$ & $1196.0 \pm 806.7$ & $1436.0 \pm 1003.1$ & $1957.3 \pm|3| 0.8$ & 0.044 \\
\hline \multicolumn{6}{|l|}{ Medications } \\
\hline$\beta$-Blockers, n (\%) & $66(77.6)$ & $20(83.3)$ & $24(66.7)$ & $22(88.0)$ & 0.106 \\
\hline CCB, n (\%) & $20(23.5)$ & $8(33.3)$ & $5(13.9)$ & $7(28.0)$ & 0.181 \\
\hline \multicolumn{6}{|l|}{ Echocardiographic parameters } \\
\hline Anomalous muscle bundle, $\mathrm{n}(\%)$ & $18(21.2)$ & $5(20.8)$ & $10(27.8)$ & $3(12.0)$ & 0.372 \\
\hline SAM, n (\%) & $75(88.2)$ & $18(75.0)$ & $32(88.9)$ & $25(100 \%)$ & 0.016 \\
\hline LVOT gradients at rest $(\mathrm{mmHg})$ & $82.5 \pm 38.3$ & $74.4 \pm 37.8$ & $81.0 \pm 41.8$ & $92.4 \pm 32.1$ & 0.246 \\
\hline LVOT gradients at provocation & $99.9 \pm 35.6$ & $110.1 \pm 49.2$ & $92.6 \pm 23.4$ & $111.0 \pm 62.2$ & $0.54 I$ \\
\hline$(\mathrm{mmHg})^{\dagger}$ & & & & & \\
\hline \multicolumn{6}{|l|}{ Cardiovascular magnetic resonance } \\
\hline LAAP diameter $(\mathrm{mm})$ & $41.3 \pm 7.0$ & $39.9 \pm 6.9$ & $42.6 \pm 6.9$ & $40.8 \pm 7.3$ & 0.316 \\
\hline LVEDD $(\mathrm{mm})$ & $45.8 \pm 5.2$ & $44.3 \pm 3.4$ & $46.0 \pm 4.8$ & $46.8 \pm 6.8$ & 0.218 \\
\hline Septal thickness (mm) & $22.0 \pm 3.4$ & $22.0 \pm 3.1$ & $22.6 \pm 3.3$ & $21.3 \pm 3.6$ & 0.313 \\
\hline LVEF $(\%)$ & $71.3 \pm 6.5$ & $71.3 \pm 6.8$ & $70.5 \pm 7.2$ & $72.7 \pm 4.9$ & 0.447 \\
\hline LVEDV index $\left(\mathrm{mL} / \mathrm{m}^{2}\right)$ & $66.3 \pm 15.4$ & $62.9 \pm 12.3$ & $67.1 \pm 16.7$ & $68.3 \pm 16.0$ & 0.431 \\
\hline LVESV index $\left(\mathrm{mL} / \mathrm{m}^{2}\right)$ & $19.3 \pm 6.9$ & $18.4 \pm 7.0$ & $20.1 \pm 7.6$ & $18.8 \pm 5.7$ & 0.613 \\
\hline SV index $\left(\mathrm{mL} / \mathrm{m}^{2}\right)$ & $47.0 \pm 10.9$ & $44.4 \pm 7.7$ & $47.1 \pm 11.9$ & $49.5 \pm 11.7$ & 0.267 \\
\hline Cardiac index $\left(1 / \mathrm{min} / \mathrm{m}^{2}\right)$ & $3.2 \pm 0.8$ & $3.0 \pm 0.7$ & $3.1 \pm 0.8$ & $3.5 \pm 1.0$ & 0.059 \\
\hline Left ventricular mass index $\left(\mathrm{g} / \mathrm{m}^{2}\right)^{\ddagger}$ & $82.0 \pm 23.4$ & $73.4 \pm 22.5$ & $81.5 \pm 22.3$ & $90.8 \pm 23.3$ & 0.031 \\
\hline LGE in ventricular septum, n (\%) & 61 (7I.8) & $17(70.8)$ & $28(77.8)$ & $16(64.0)$ & 0.497 \\
\hline
\end{tabular}

Notes: Values are mean \pm SD or $n(\%)$. Group A defined as initial r-wave in bothleads VI and aVR, $n=24$; Group $B$ defined as initial r-wave in lead VI but not in lead aVR, $\mathrm{n}=36$; and Group $C$ defined as without initial $r$-wave in both leads of VI or aVR, $n=25$. *NT-proBNP (fmol/mL): LSD $t$-test showed the difference between Group $A$ and Group B ( $p=0.384)$, Group A and Group C ( $p=0.014)$, and Group B and Group $C(p=0.067)$. ${ }^{\dagger}$ LVOT gradients was provoked in 22 of the 85 patients. ${ }^{\ddagger}$ Left ventricular mass index $\left(\mathrm{g} / \mathrm{m}^{2}\right)$ : LSD $t$-test showed the difference between Group A and Group B ( $p=0.178$ ), Group A and Group $C$ ( $\left.p=0.009\right)$, and Group B and Group $C$ ( $\left.p=0.118\right)$. Abbreviations: SBP, systolic blood pressure; DBP, diastolic blood pressure; NYHA, New York Heart Association; HOCM, hypertrophic obstructive cardiomyopathy; SCD, sudden cardiac death; VT, ventricular tachycardia; NT-proBNP, N-terminal pro-brain natriuretic peptide, CCB, calcium channel blocker; SAM, systolic anterior motion; LVOT, left ventricular outflow tract; LAAPD; left atrial anteroposterior diameter; LVEDD; left ventricular end-diastolic dimension; LVEF, left ventricular ejection fraction; LVEDV, left ventricular end-diastolic volume; LVESV, left ventricular end-systolic volume; SV, stroke volume; LGE, late gadolinium enhancement.

between with and without changes in initials r-wave in leads V1 or aVR, before PTSMA procedure there were significant differences in the left ventricular mass index and none in LVOT gradients at rest, but after the PTSMA procedure there were significant differences in residual gradients only in lead aVR but not in V1. 
Table 2 PTSMA Procedure-Related Profiles and 3-Year Follow-Up

\begin{tabular}{|c|c|c|c|c|c|}
\hline & Total Population $(n=85)$ & Group A $(n=24)$ & Group B $(n=36)$ & Group $C(n=25)$ & $p$ value \\
\hline Ethanol volume $(\mathrm{mL})$ & $2.1 \pm 0.8$ & $2.3 \pm 0.9$ & $2.0 \pm 0.8$ & $2.2 \pm 0.8$ & 0.248 \\
\hline Number of ablated branches, $\mathrm{n}$ & $1.0 \pm 0.0$ & $1.0 \pm 0.0$ & $1.0 \pm 0.0$ & $1.0 \pm 0.0$ & 1.000 \\
\hline Invasive gradient pre-PTSMA (mmHg) & $98.5 \pm 44.8$ & $101.0 \pm 48.6$ & $92.6 \pm 43.8$ & $104.4 \pm 43.3$ & 0.575 \\
\hline Invasive gradient post-PTSMA (mmHg) & $32.0 \pm 29.3$ & $29.5 \pm 15.6$ & $29.5 \pm 30.8$ & $37.9 \pm 36.6$ & 0.492 \\
\hline Acute success, $n(\%)^{*}$ & 74(87.1) & $23(95.8)$ & $32(88.9)$ & $19(76)$ & 0.107 \\
\hline \multicolumn{6}{|c|}{ Within I week post-PTSMA procedure } \\
\hline Residual gradient $(\mathrm{mmHg})^{\dagger}$ & $47.0 \pm 32.9$ & $39.4 \pm 36.0$ & $50.5 \pm 34.9$ & $49.8 \pm 26.0$ & 0.404 \\
\hline New occurred RBBB, $n(\%)$ & $47(55.3)$ & II(45.8) & $20(55.6)$ & $16(64.0)$ & $0.44 I$ \\
\hline Initial $r$-wave in $V_{1}, n(\%)$ & $28(32.9)$ & $\mathrm{II}(45.8)$ & $14(38.9)$ & $3(12.0)$ & 0.025 \\
\hline Initial $r$-wave in aVR, $n(\%)$ & $4(4.7)$ & $4(16.7)$ & $0(0.0)$ & $0(0.0)$ & 0.005 \\
\hline Changes in initial $r$-wave in $V_{1}, n(\%)$ & $38(44.7)$ & $13(54.2)$ & $22(6 I .1)$ & $3(12.0)$ & 0.000 \\
\hline Changes in initial r-wave in aVR, $n(\%)$ & $20(23.5)$ & $20(83.3)$ & $0(0.0)$ & $0(0.0)$ & 0.000 \\
\hline \multicolumn{6}{|l|}{ Follow-up } \\
\hline$\beta$-Blockers, $n(\%)^{\ddagger}$ & $21(24.7)$ & $4(16.7)$ & II(30.6) & $6(24.0)$ & 0.472 \\
\hline Primary outcome, $\mathrm{n}(\%)$ & $22(25.8)$ & $2(8.3)$ & $10(27.7)$ & $10(40.0)$ & 0.038 \\
\hline Death, $n(\%)$ & $0(0)$ & $0(0)$ & $0(0)$ & $0(0)$ & - \\
\hline Surgical myectomy, n(\%) & $9(10.6)$ & $\mathrm{I}(4 . \mathrm{I})$ & $4(11.1)$ & $4(16.0)$ & - \\
\hline Syncope, $n(\%)$ & $0(0)$ & $0(0)$ & $0(0)$ & $0(0)$ & - \\
\hline NYHA classification III/IV, n(\%) & $13(15.2)$ & $\mathrm{I}(4 . \mathrm{I})$ & $6(16.6)$ & $6(24.0)$ & - \\
\hline
\end{tabular}

Notes: Group A defined as initial r-wave in both leads VI and aVR, n=24; Group B defined as initial r-wave in lead VI but not in lead aVR, $n=36$; and Group C defined as without initial $r$-wave in both leads $V I$ and $a V R, n=35$. *Acute success was defined more than $50 \%$ reduction of invasive gradient pre-PTSMA, or less than $30 \mathrm{mmHg}$ of invasive gradient post-PTSMA. ${ }^{\dagger}$ Residual gradient: within 7 days after PTSMA, all the patients had been performed transthoracic echocardiography for measuring residual gradient. ${ }^{\ddagger} \beta$-Blockers: during 3-year follow-up.

Abbreviation: PTSMA, percutaneous transluminal septal myocardial ablation.

\section{Cumulative Incidence of Primary Outcomes}

During 3-year follow-up, there was no significant difference concerning taking $\beta$-blockers. There were 9 (10.6\%) patients with surgical myectomy, 13 (15.3\%) patients with NYHA classification III/IV, and 37 $(43.5 \%)$ patients with cardiovascular symptomatic. Univariable and multivariable Cox analyses identified independent predictors of the primary outcomes in Table 3. Compared with without initial r-wave in leads $\mathrm{V} 1$ and aVR, initial r-wave in both leads V1 and aVR before PTSAM were independently associated with a decreased risk of primary outcomes (HR: $0.112 ; 95 \%$ CI: 0.024 to $0.518 ; p=0.005$ ) in patients with HOCM during 3 years after PTSMA (Table 3). In Figure 3, Kaplan-Meier curves with cumulative hazards of primary outcomes compared according to the presence of initials r-wave in leads V1 and aVR, changes of the presence of initial $\mathrm{r}$-wave in lead V1, and changes of the presence of initial $r$-wave in lead aVR

\section{Discussion}

The main and novel finding of our study is that, before PTSMA, the presence of initial r-wave in QRS waves of leads V1 and aVR on the surface 12-lead ECG is highly predictive of good mid-term response to PTSMA in patients with HOCM.

Sigwart was the first to report a successful nonsurgical myocardial reduction in cases of HOCM through occlusion of the septal branch using pure alcohol. ${ }^{15}$ PTSMA aims to achieve a reduction in septal muscular mass through a limited therapeutic infarction, thus reducing the extent of the outflow tract obstruction. Several registries have demonstrated that PTSMA could improve mid-term prognosis and is an established treatment for resolving heart failure-associated symptoms in HOCM. ${ }^{16,17}$ There were no patients occurred with death during a 3-year follow-up in our study population. But from Figure 1, there was one patient death for peri-procedural complication, and the mortality was about $1 \%$ (1 in 100 patients) in patients who underwent PTSMA, which was near to 

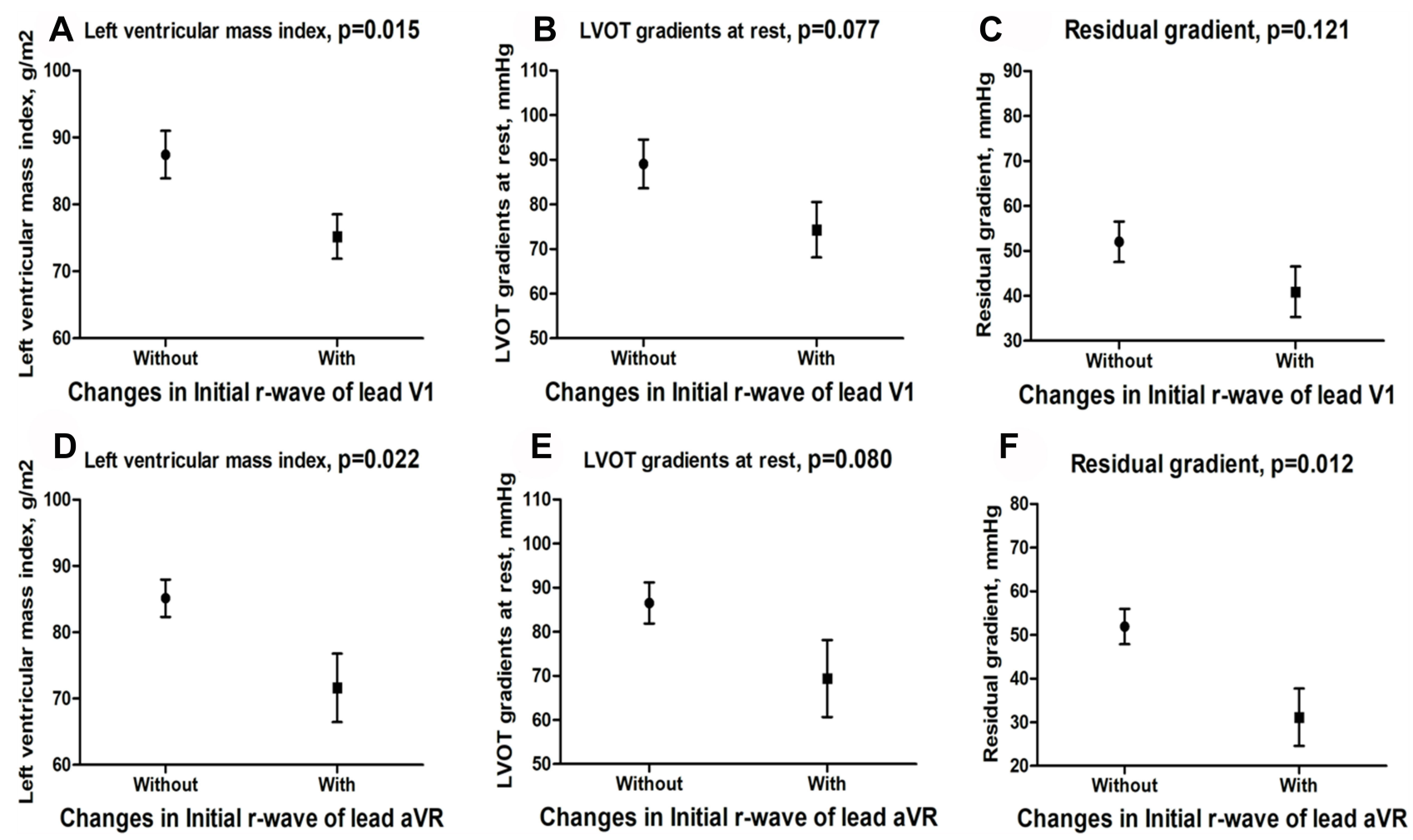

Figure 2 Compared the left ventricular mass index (A and D), LVOT gradients at rest (B and $\mathbf{E})$ and LVOT residual gradients (C and $\mathbf{F})$ according to the presence of initials $r$-wave in leads VI \& aVR.

Angelika's study. They reported that 952 patients underwent PTSMA, and 70 patients died during 5-year followup. $^{18}$

Satisfactory results were not observed in all patients who underwent PTSMA. It is important to identify the determinants of PTSMA outcome for better selection of patients and potential modification of treatment plan in an individual patient. Chang's study ${ }^{5}$ has demonstrated that the residual LVOT gradient and peak CK leak after PTSMA are the independent predictors of PTSMA outcomes, and all of age, septal thickness, mitral regurgitation, baseline gradient, and ventricular function before PTSMA procedure were not determinants of PTSMA outcome. The main limitation of Chang's study is that these predictors, included residual LVOT and peak CK, were only to be known after the PTSAM procedure. In our study, we did not analyze the data of peak CK leak after PTSMA, and the residual LVOT gradient was significantly different when grouped by the presence of primary outcome (Figure S1). Steggerda et $\mathrm{al}^{19}$ had demonstrated that baseline gradient was associated with an unsuccessful outcome in 4 months follow-up after PTSMA, which was uncompatible with Chang's study. We also did not find that the baseline gradient was the predictor of PTSMA outcome during 3-year follow-up. The possible reason is that the follow-up time of Steggerda's study was short.

Initial r-wave in both leads $\mathrm{V} 1$ and aVR before PTSMA is an independent predictor of good midterm PTSMA outcome in our study. The mechanism of this phenomenon was unknown. The initial r-wave in both leads V1 and aVR is the sign of depolarization of the ventricular septal base. As shown in Table 1, before PTSMA, the presence of initial r-wave in leads V1 and aVR was associated with the presence of SAM phenomenon, left ventricular mass index, and level of NT-proBNP. Patients without the presence of initial r-wave in both leads V1 and aVR had worse cardiac function (NYHA classification III/IV). After PTSMA procedural, the most basal part of the septum may be spared causing residual obstruction in some patients, ${ }^{20}$ and the benefits of PTSMA depend on electrical effects, which involve both main branches of the bundle of His and loss of septal r-wave, as well as on the extent of septal myocardial infarction. ${ }^{6}$ A case report published in 1991 described the ECG changes in pure septal infarct resulting from the iatrogenic occlusion of the first septal branch of the left anterior 
Table 3 Univariate and Multivariate Cox Analysis for Primary Outcome

\begin{tabular}{|c|c|c|}
\hline & \multicolumn{2}{|c|}{ Primary Outcomes } \\
\hline & HR (95\% Cl) & $p$ value \\
\hline \multicolumn{3}{|c|}{ Model I } \\
\hline Group A $(n=24)$ & $0.120(0.026-0.554)$ & 0.007 \\
\hline Group B $(n=36)$ & $0.465(0.192-1.127)$ & 0.090 \\
\hline Group $C(n=25)$ & Ref & Ref \\
\hline \multicolumn{3}{|c|}{ Model 2} \\
\hline Group A $(n=24)$ & $0.120(0.026-0.554)$ & 0.007 \\
\hline Group B $(n=36)$ & $0.465(0.192-1.127)$ & 0.090 \\
\hline Group C $(n=25)$ & Ref & Ref \\
\hline \multicolumn{3}{|c|}{ Model 3} \\
\hline Group A $(n=24)$ & $0.1 I 2(0.024-0.5 \mid 8)$ & 0.005 \\
\hline Group B $(n=36)$ & $0.533(0.217-1.310)$ & 0.170 \\
\hline Group C $(n=25)$ & Ref & Ref \\
\hline
\end{tabular}

Notes: Group A defined as initial $r$-wave in both leads VI and aVR, $n=24$; Group $B$ defined as initial $r$-wave in lead VI but not in lead aVR, $n=36$; and Group $C$ defined as without initial $r$-wave in both leads of $\mathrm{VI}$ or $\mathrm{aVR}, \mathrm{n}=25$. Primary outcomes included death, surgical myectomy, syncope and NYHA classification III/IV. Model I: unadjusted. Model 2: multivariate adjustment was made for age, sex, and NYHA class III/IV. Model 3: multivariate adjustment was made for age, sex, NYHA class III/ IV, hypertension, NT-proBNP, SAM and left ventricular mass index.

Abbreviation: HR hazard ratio.

descending coronary artery during percutaneous transluminal coronary angioplasty. Compared to the pre-procedure ECG, the post-procedure ECG showed new ST-segment elevation in leads V1 and disappearance of r-wave in leads V1. ${ }^{21}$ As concluded for Figures 2 and 3, the changes of the presence of initial r-wave, especially in lead aVR, were associated with left ventricular mass index and LVOT residual gradients. Loss of the septal $r$ wave was associated with septal asynchrony, whose most obvious characteristic is post-ejection shortening. ${ }^{22}$ In our study, compared with none initial r-wave in both leads V1 and aVR or initial $\mathrm{r}$-wave only in lead V1, the presence of initial r-wave in both leads V1 and aVR on the surface 12lead ECG is highly predictive of good mid-term response to PTSMA in patients with HOCM (primary outcomes, HR: $0.112 ; 95 \%$ CI: 0.024 to $0.518 ; p=0.005)$. We speculated that the PTSMA procedural maybe could be more effective to improve the cardiac function during midterm follow-up in HOCM patients with the presence of initial r-wave in both leads V1 and aVR, through conduction disturbances of the interventional septal base, not just the limited myocardial infarction. And in HOCM patients without initial r-wave in leads $\mathrm{V} 1$ and/or aVR, maybe the early activation of septal basal was not the main cause for worse cardiac function after PTSMA procedure.

However, the presence of initial r-wave in QRS waves of leads V1 and aVR was not predictive of acute success response to PTSMA in patients with HOCM, maybe due to delayed electrical reconstruction and the ongoing reduction of the SAM phenomenon in further follow-up. ${ }^{23}$

There are some limitations in our study. First, we defined NYHA classification III/IV and surgical myectomy as primary outcomes because there were no patients occurred with death, syncope, pacemaker, or stroke, and not every patient underwent echocardiography for measuring LVOT gradient during 3-year follow-up. Since NYHA III/IV symptoms persistence would have likely led to the surgical myectomy, it is possible
A Grouped by presence of Initial r-wave, Log-rank $p=0.006$

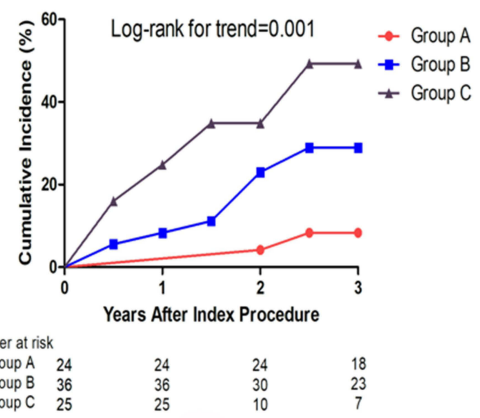

B Changes in Initial r-wave of lead V1, Log-rank $\mathrm{p}=0.447$

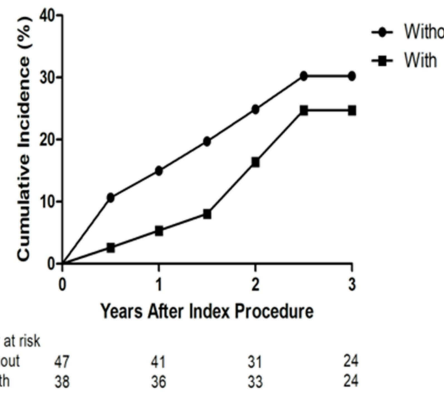

C Changes in Initial r-wave of lead aVR, Log-rank $\mathrm{p}=0.002$

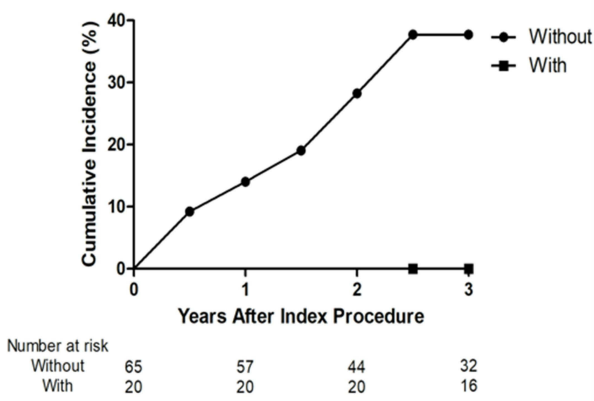

Figure 3 Cumulative incidence of primary outcomes. Kaplan-Meier curves with cumulative hazards of primary outcomes compared according to the presence of initials $r$-wave in leads VI and aVR (A), changes of the presence of initial r-wave in lead VI (B), and changes of presence of initial r-wave in lead aVR. Primary outcomes included surgical myectomy (Morrow surgery) and NYHA classification III/IV (C). Group A defined as initial r-wave in both leads VI and aVR; Group B defined as initial r-wave in lead VI but not in lead aVR; Group $C$ defined as without initial $r$-wave in both leads VI and aVR. 
that conclusion was been over accounted for. However, previous studies to evaluate outcomes in patients who have undergone PTSMA, mostly using functional assessment by NYHA class with 3-year follow-up, have shown that the method is safe and effective. ${ }^{24-26}$ Second, this was a singlecenter study with a relatively small sample size. But, the difference between groups is significant and obvious.

\section{Conclusions}

The results suggest that the presence of initial r-wave in both leads V1 and aVR on the surface 12-lead ECG is highly predictive of good mid-term response to PTSMA in patients with HOCM.

\section{Data Sharing Statement}

All data are freely available with reasonable requirements from authors.

\section{Funding}

There is no funding to report.

\section{Disclosure}

The authors report no conflicts of interest in this work.

\section{References}

1. Ho CY, Lopez B, Coelho-Filho OR, et al. Myocardial fibrosis as an early manifestation of hypertrophic cardiomyopathy. $N$ Engl J Med. 2010;363:552-563. doi:10.1056/NEJMoa1002659

2. Steggerda RC, Damman K, Balt JC, et al. Periprocedural complications and long-term outcome after alcohol septal ablation versus surgical myectomy in hypertrophic obstructive cardiomyopathy. JACC Cardiovasc Interv. 2014;7(11):1227-1234. doi:10.1016/j.jcin.201 4.05 .023

3. Faber L, Welge D, Fassbender D, et al. One-year follow-up of percutaneous septal ablation for symptomatic hypertrophic obstructive cardiomyopathy in 312 patients: predictors of hemodynamic and clinical response. Clin Res Cardiol. 2007;96(12):864-873. doi:10.1007/ s00392-007-0578-9

4. Maekawa Y, Jinzaki M, Anzai A, et al. Successful second attempt multidetector computed tomography-guided percutaneous transluminal septal myocardial ablation for an octogenarian with hypertrophic obstructive cardiomyopathy. Int J Cardiol. 2014;176(3):e131-e132. doi:10.1016/j.ijcard.2014.07.211

5. Chang SM, Lakkis NM, Franklin J, et al. Predictors of outcome after alcohol septal ablation therapy in patients with hypertrophic obstructive cardiomyopathy. Circulation. 2004;109(7):824-827. doi:10.1161/ 01.CIR.0000117089.99918.5A

6. Henein MY, O'Sullivan CA, Ramzy IS, et al. Electromechanical left ventricular behavior after nonsurgical septal reduction in patients with hypertrophic obstructive cardiomyopathy. J Am Coll Cardiol. 1999;34 (4):1117-1122. doi:10.1016/S0735-1097(99)00332-0

7. Kappenberger L, Linde C, Daubert C, et al. Pacing in hypertrophic obstructive cardiomyopathy. A randomized crossover study. PIC Study Group. Eur Heart J. 1997;18:1249-1256. doi:10.1093/oxfordjournals. eurheartj.a015435
8. Spach MS, Barr RC. Ventricular intramural and epicardial potential distributions during ventricular activation and repolarization in the intact dog. Circ Res. 1975;37:243-257. doi:10.1161/01.RES.37.2.243

9. Durrer D, van Dam RT, Freud GE, et al. Total excitation of the isolated human heart. Circulation. 1970;41(6):899-912. doi:10.1161/01.CIR.41.6.899

10. Gurjarpadhye A, Hewett KW, Justus C, et al. Cardiac neural crest ablation inhibits compaction and electrical function of conduction system bundles. Am J Physiol Heart Circ Physiol. 2007;292(3): H1291-H1300. doi:10.1152/ajpheart.01017.2006

11. Elliott PM, Anastasakis A, Borger MA, et al. 2014 ESC guidelines on diagnosis and management of hypertrophic cardiomyopathy: the task force for the diagnosis and management of hypertrophic cardiomyopathy of the European Society of Cardiology (ESC). Eur Heart J. 2014;35:2733-2779.

12. Nagueh SF, Bierig SM, Budoff MJ, et al. American society of echocardiography clinical recommendations for multimodality cardiovascular imaging of patients with hypertrophic cardiomyopathy: endorsed by the American society of nuclear cardiology, society for cardiovascular magnetic resonance, and society of cardiovascular computed tomography. J Am Soc Echocardiogr. 2011;24:473-498. doi:10.1016/j.echo.2011.03.006

13. Zhang C, Liu R, Yuan J, et al. Significance and determinants of cardiac troponin I in patients with obstructive hypertrophic cardiomyopathy. Am J Cardiol. 2015;116(11):1744-1751. doi:10.1016/j.amjcard.2015.09.006

14. Bennett JA, Riegel B, Bittner V, et al. Validity and reliability of the NYHA classes for measuring research outcomes in patients with cardiac disease. Heart Lung. 2002;31(4):262-270. doi:10.1067/ mhl.2002.124554

15. Sigwart U. Non-surgical myocardial reduction for hypertrophic obstructive cardiomyopathy. Lancet. 1995;346(8969):211-214. doi:10.1016/S0140-6736(95)91267-3

16. Sorajja P, Ommen SR, Holmes DJ, et al. Survival after alcohol septal ablation for obstructive hypertrophic cardiomyopathy. Circulation. 2012;126(20):2374-2380. doi:10.1161/CIRCULATIONAHA.111.07 6257

17. Veselka J, Krejci J, Tomasov P, et al. Long-term survival after alcohol septal ablation for hypertrophic obstructive cardiomyopathy: a comparison with general population. Eur Heart J. 2014;35:2040-2045. doi:10.1093/eurheartj/eht495

18. Batzner A, Pfeiffer B, Neugebauer A, et al. Survival after alcohol septal ablation in patients with hypertrophic obstructive cardiomyopathy. J Am Coll Cardiol. 2018;72(24):3087-3094. doi:10.1016/j.jacc.2018.09.064

19. Steggerda RC, Balt JC, Damman K, et al. Predictors of outcome after alcohol septal ablation in patients with hypertrophic obstructive cardiomyopathy. Special interest for the septal coronary anatomy. Neth Heart J. 2013;21(11):504-509. doi:10.1007/ s12471-013-0453-4

20. Valeti US, Nishimura RA, Holmes DR, et al. Comparison of surgical septal myectomy and alcohol septal ablation with cardiac magnetic resonance imaging in patients with hypertrophic obstructive cardiomyopathy. $J$ Am Coll Cardiol. 2007;49(3):350-357. doi:10.1016/j.jacc.2006.08.055

21. Tamura A, Kataoka H, Mikuriya Y. Electrocardiographic findings in a patient with pure septal infarction. Br Heart J. 1991;65:166-167. doi:10.1136/hrt.65.3.166

22. Xiao HB, Gibson DG. Absent septal q wave: a marker of the effects of abnormal activation pattern on left ventricular diastolic function. Br Heart J. 1994;72:45-51. doi:10.1136/hrt.72.1.45

23. Seggewiss H, Gleichmann U, Faber L, et al. Percutaneous transluminal septal myocardial ablation in hypertrophic obstructive cardiomyopathy: acute results and 3-month follow-up in 25 patients. $J \mathrm{Am}$ Coll Cardiol. 1998;31(2):252-258. doi:10.1016/S0735-1097(97) 00508-1 
24. Ruzyllo W, Chojnowska L, Demkow M, et al. Left ventricular outflow tract gradient decrease with non-surgical myocardial reduction improves exercise capacity in patients with hypertrophic obstructive cardiomyopathy. Eur Heart J. 2000;21:770-777. doi:10.1053/ euhj.1999.1905

25. Chojnowska L, Ruzyllo W, Witkowski A, et al. Early and long-term results of non-surgical septal reduction in patients with hypertrophic cardiomyopathy. Kardiol Pol. 2003;59:269-282.
26. Kim JJ, Lee CW, Park SW, et al. Improvement in exercise capacity and exercise blood pressure response after transcoronary alcohol ablation therapy of septal hypertrophy in hypertrophic cardiomyopathy. Am J Cardiol. 1999;83:1220-1223. doi:10.1016/ S0002-9149(99)00063-6

\section{Publish your work in this journal}

The International Journal of General Medicine is an international, peer-reviewed open-access journal that focuses on general and internal medicine, pathogenesis, epidemiology, diagnosis, monitoring and treatment protocols. The journal is characterized by the rapid reporting of reviews, original research and clinical studies across all disease areas. The manuscript management system is completely online and includes a very quick and fair peer-review system, which is all easy to use. Visit http://www.dovepress.com/ testimonials.php to read real quotes from published authors. 